\title{
Introduction to special section: Skeletonized/sparse/multiscale geophysical inversion for the interpreter
}

Gaurav Dutta ${ }^{1}$, Tristan van Leeuwen ${ }^{2}$, Amr Ibrahim ${ }^{3}$, Alexander Klokov ${ }^{4}$, Yunsong Huang ${ }^{5}$

Seismic inversion methods are mostly based on gradient-based optimization techniques that minimize the data residual between the observed and the predicted data, which is usually computed from a given estimate of the model. This conventional optimization problem can be reformulated as inversion of skeletonized data, sparse inversion, or multiscale inversion that share the same aim of finding useful solutions by reducing the complexity of either the data space and/or the model space and yet, still use the fundamental governing equations without the need for approximations.

Skeletonized inversion relies on using simplified data sets derived from the original data that retain the important information about the model parameter of interest. Examples of skeletonized data sets include first-arrival traveltimes picked for velocity inversion; amplitudes of transmitted earthquake SH-waves for earthquake moment inversion; frequency-shifts measured from first arrivals for $\mathrm{Q}$ tomography or picked dispersion curves for surface-wave tomography. Sparse inversion, on the other hand, aims to invert for a simplified (sparse) representation of the data/model in some transform domain like the Radon, wavelet or curvelet-domains. Multiscale inversion iteratively inverts the data at different scales of the model and data, usually starting with the long wavelength scale and ending at a shorter wavelength scale.

This special section is devoted to understanding the theory, the opportunities and the limitations of skeletonized/sparse/multiscale geophysical inversion by highlighting some applications of these techniques on synthetic and field seismic data.

Fu and Sherif present examples of applying the wave equation traveltime tomography (WT) and ray-tracing traveltime tomography (RT) methods to near-surface seismic land data. Results show that WT can provide a more accurate tomogram than RT. In addition, WT is also less sensitive to the additive noise in the input data than RT.

Feng and Schuster use skeletonized inversion to estimate the velocity and anisotropy parameters in a transversely isotropic medium with a vertical symmetry axis (VTI medium). They validate the effectiveness of their proposed approach on synthetic data examples and a marine data set recorded in the Gulf of Mexico.

Lu et al., present a mostly non-mathematical tutorial that explains the theory of wave-equation inversion of skeletonized data. They use four examples to demonstrate some of the applications of skeletonized inversion.

\footnotetext{
${ }^{1}$ CGG, Houston, Texas, USA. Email: gaurav.dutta@cgg.com.

${ }^{2}$ Utrecht University, Utrecht, Netherlands. Email: T.vanLeeuwen@uu.nl.

${ }^{3}$ King Abdullah University of Science and Technology, Thuwal, Saudi Arabia. Email: amr.ibrahim@kaust.edu.sa.

${ }^{4}$ University of Texas, Austin, USA. Email: alexander.klokov@gmail.com.

${ }^{5}$ Los Alamos National Laboratory, New Mexico, USA. Email: yunsongh@gmail.com. 